\title{
Mucormycosis in a diabetic ketoacidosis patient
}

\author{
G. Sree Vijayabala ${ }^{1 *}$, Rajeshwari G Annigeri ${ }^{2}$, Ramachandran Sudarshan ${ }^{3}$ \\ ${ }^{1}$ Department of Dentistry, ESIC Medical, College and PGIMSR, K.K. Nagar, Chennai, India \\ ${ }^{2}$ Department of Oral Medicine and Radiology, College of Dental Sciences \& Hospital, Davangere, India \\ ${ }^{3}$ Department of Oral Medicine and Radiology, Best Dental Science College, Madurai, India
}

\section{PEER REVIEW}

Peer reviewer

Malinee Pongsavee, Ph.D., Associate Professor, Department of Medical Technology, Faculty of Allied Health Sciences, Thammasat University, Rangsit Campus Patumthani 12121, Thailand.

Tel: 662-9869213 ext.7252

Fax: 662-5165379

E-mail : malineep@tu.ac.th

Comments

This is a good case report about mucormycosis in diabetic ketoacidosis patient. It may apply for the treatment in the other opportunistic fungal infection. Details on Page 833

\section{ABSTRACT}

Oral cavity is considered to be a kaleidoscope for body's general health. Many systemic conditions do present with diverse oral manifestations. Mucormycosis involving the oral cavity is one such entity that presents as necrosis of bone in immunocompromised patients. Mucormycosis is an opportunistic fungal infection that mainly affects the patients with uncontrolled diabetes mellitus. Hereby, we report a case of mucormycosis involving the palate in a patient with diabetic ketoacidosis.
KEYWORDS

Mucormycosis, Diabetic ketoacidosis, Palatal necrosis

\section{Introduction}

Mucormycosis is an aggressive, frequently fatal invasive fungal infection that can develop in immunocompromised patients[1]. It is usually documented in patients with uncontrolled diabetes. Mucor is often recognized as a triad of symptoms, such as uncontrolled diabetes mellitus, periorbital infection and meningoenchephalitis[2].

\section{Case report}

A 50 years old male patient, working as a farmer reported with the chief complaint of pain in the left upper back teeth region since $25 \mathrm{~d}$ which was sudden, severe, throbbing and continuous. It was localized to the particular region and is aggravated on taking foods with inability to brush. The patient had a history of stroke $25 \mathrm{~d}$ before following which he developed blindness in left eye since $20 \mathrm{~d}$. He reported of swelling of left middle face and difficulty in breathing with pus discharge from nose since $15 \mathrm{~d}$. Patient had spontaneous exfoliation of left upper back teeth $10 \mathrm{~d}$ before. History also revealed that the patient had frequent giddiness since 3 years and fever with chills for past 1 month. Patient was recently diagnosed to have diabetes 1 month before when he was admitted to the hospital for

Article history:

Received 28 Jul 2013

Received in revised form 10 Aug, 2nd revised form 19 Aug, 3rd revised form 5 Sep 2013 Accepted 10 Sep 2013

Available online 28 Otc 2013 
stroke.

Medical history revealed that patient had Type 2 diabetes mellitus with diabetes ketoacidosis (diagnosed a month before) and he was also a hypertensive since 5 years. Drug history revealed that patient was on injection insulin $(10 \mathrm{U}-10 \mathrm{U}-8 \mathrm{U} /$ day $)$ and injecion amphotericin B (1 vial in DNS/day) since a month.

Extraoral examination revealed a solitary swelling in the left side of the face obliterating the nasolabial fold and orbital cellulitis with pus discharge. Blackish mucopus was seen extruding from the left nose. On intraoral examination, maxillary palatal necrosis of about $4.5 \times 5 \mathrm{~cm}$ was seen extending from 24 to 28 region (Figure 1). There was gingival and mucosal denudation exposing the necrotic bone with pus exudation. Extreme tenderness was elicited on palpation. Hard tissue examination revealed missing 27 with mobility and tenderness on percussion in 24, 25, 26, and 28. Based on these findings, a provisional diagnosis of mucormycosis involving the palate was given with a differential diagnosis of Wegener's granulomatosis being considered. An array of investigations was carried out. His blood sugar level was $545 \mathrm{mg} / \mathrm{dl}$ before a month and now under control. Occlusal and panoramic view showed complete bone loss in distal aspect of 26 (Figure 2 and 3). CT of Paranasal Sinuses depicted mucosal thickening in left frontal, posterior ethmoid, sphenoid and floor of right maxillary sinus. Left maxillary sinus showed fluid level with bubbly appearance and mucosal thickening obliterating left osteomeatal opening. CT of brain was normal. Tissue biopsy with histopathology showed non septate mucormycotic hypae at 90 degrees. Klebsiella and candida were detected in pus culture (Figure 4).

Under General anesthesia, palatal debridement was done with extraction of 28 (Figure 5). Topical rinsing with hydrogen peroxide and povidone iodine was advised with local amphotericin $\mathrm{B}$ instillation of $3 \mathrm{~mL}$ and the patient was advised to continue injection insulin and amphotericin B.

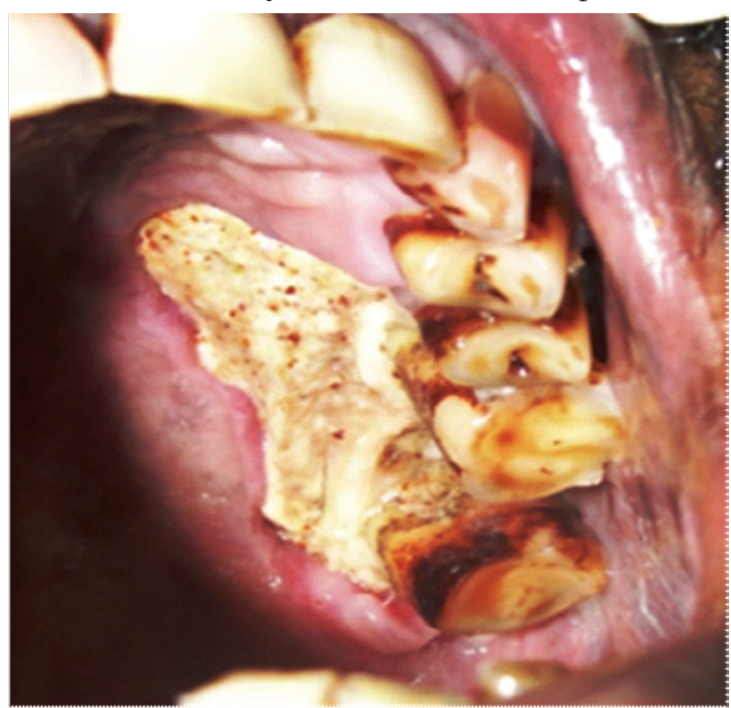

Figure 1. Maxillary palatal necrosis of about $4.5 \times 5 \mathrm{~cm}$ was seen extending from 24 to 28 region.

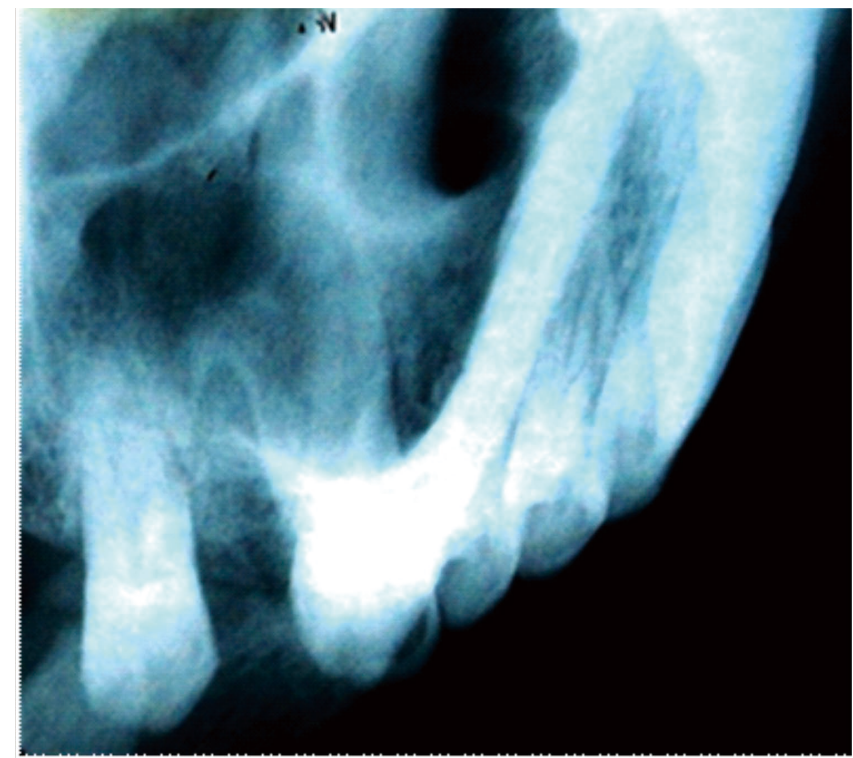

Figure 2. Occlusal radiograph revealing complete bone loss in distal aspect of 26

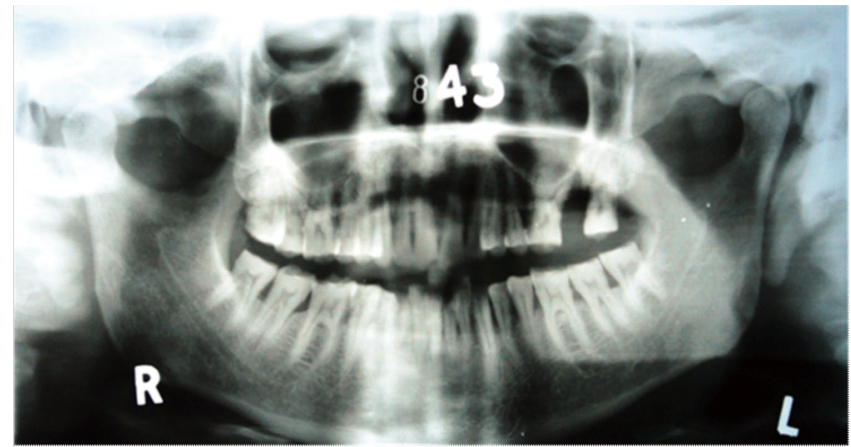

Figure 3. Panoramic view revealing complete bone loss in distal aspect of 26.

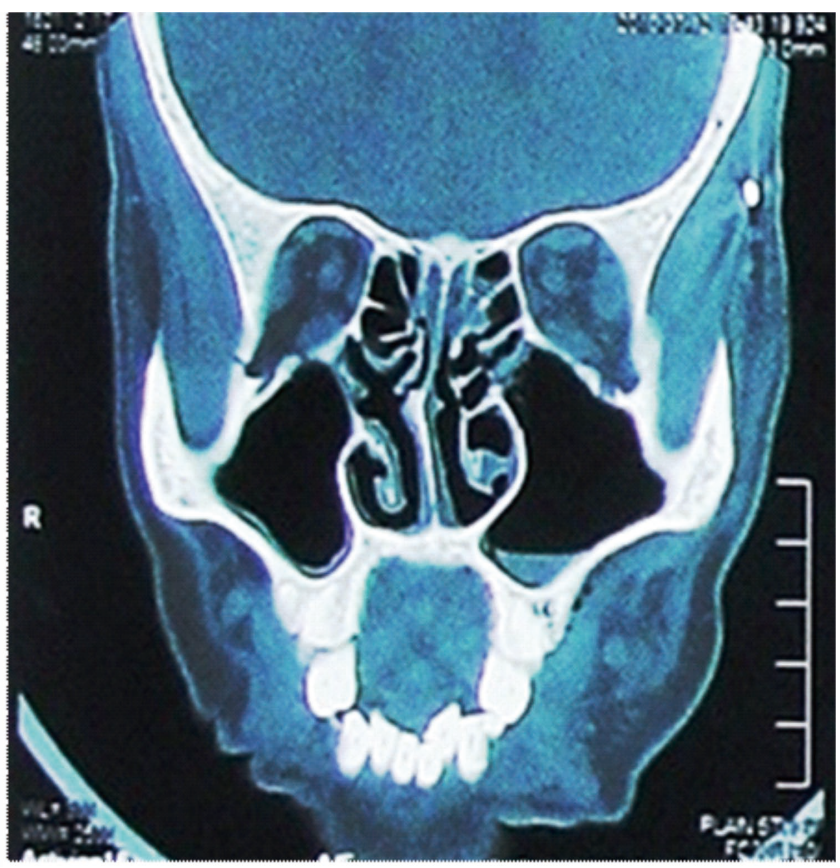

Figure 4. Computed tomography showing fluid filled left maxillary sinus. 


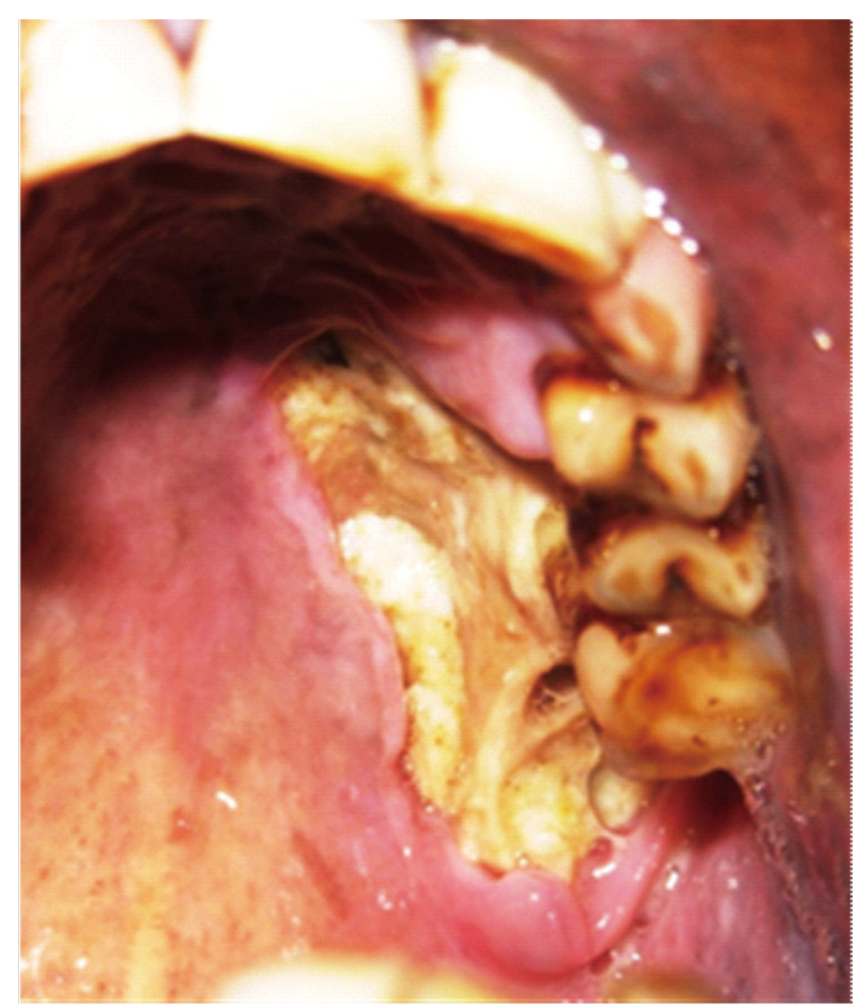

Figure 5. Post operative phase after debridement

\section{Discussion}

Mucormycosis (Zygomycosis, phycomycosis) is an acute opportunistic infection caused by a saprophytic fungus that belongs to the class of phycomycetes. Although several genera are associated with this disease, the most common forms are Rhizopus, Rhizomucor and Absida. Rhizopus is the predominant pathogen accounting for $90 \%$ of the cases of rhinocerebral mucormycosis[3]. Other fungal genera associated with the disease would include (Cunninghamella, Saksenaea, Syncephalastrum, Cokeromyces, Mortierella)[4]. Mucormycosis is classified according to the anatomic site of occurrence in (1) rhinocerebral, (2) pulmonary, (3) cutaneous, (4) gastrointestinal, and (5) disseminated[5].

Mucormycosis is aggressive and potentially fatal in diabetic patients because of impaired host defense mechanism and increased availability of micronutrients such as iron[6]. The most important conditions predisposing to mucormycosis, according to various studies, include malignant haematological disease with or without stem cell transplantation, prolonged and severe neutropenia, poorly controlled diabetes mellitus with or without diabetic ketoacidosis, iron overload, major trauma, prolonged use of corticosteroids, illicit intravenous drug use, neonatal prematurity and malnourishment[7]. In rhinocerbral mucormycosis, the infected nasal mucosa may appear normal in earliest stage, then progresses through an erythematous phase, with or without edema, followed by violaceous appearance, and finally the development of black, necrotic nasal or palatal eschar as blood vessels become thrombosed with ensuing tissue necrosis[8].

Zygomycosis of the oral cavity can be of two origins. One is from disseminated infection where the portal of entry is by inhalation (usually through the nose); the other is from direct wound contamination with dissemination to other viscera as a common complication. When arising from the nose and paranasal sinuses, the infection may cause palatal ulceration progressing to necrosis. The area appears black in the large majority of the cases. When the infection spreads from direct wound contamination, the clinical findings may appear anywhere in the oral cavity, including the mandible. An important prognostic difference between infection involving the maxilla and infection of the mandible is cavernous sinus thrombosis, a serious complication of maxillary infections[9].

Radiographic findings are generally inconclusive and not specific. CT and MRI are within normal limits initially, which are followed by signs of sinusitis, such as congested sinus or thickened mucosal lining. Repetition of the investigations is necessary for close follow-up of the advancement of the disease[10].

Differential diagnosis mainly includes necrotizing fasciitis; especially if facial edema is present[11]. The differential diagnosis of such a peculiar condition should initially include cocaine abuse, fasciitis, or other opportunistic infections of an immunocompromised host, such as aspergillosis, herpes simplex, or herpes zoster[10].

Early diagnosis is of prime importance in treating patients with mucormycosis. Correcting or controlling predisposing problems is also essential for improving the treatment outcome. In diabetic ketoacidotic patients, hyperglycemia and acidemia should be corrected[12]. Medical management includes antifungal treatment with high doses of Amphotericin B[6]. Recently intravenous liposomal Amphotericin, intravenous lipid complex and Hyperbaric oxygen therapy has also been used[13]. Early surgical excision of the infected sinuses and appropriate debridement of the retro-orbital space can often prevent the infection from extending into the eye. Repeated surgical debridement may be necessary to ensure that all necrotic tissue has been debrided and the infection has not progressed[12].

\section{Conclusion}


With the change of life style and food habits in the recent decades, there has been an alarming increase in the prevalence of diabetes worldwide. Mucormycosis of oral cavity could be seen in patients with uncontrolled diabetes mellitus as in our case. Hence, a proper understanding of such a fulminate entity is needed as the underlying systemic conditions at many times may be quiescent and dentists may be the first person to identify the debilitated status of the patient.

\section{Conflict of interest statement}

We declare that we have no conflict of interest.

\section{Comments}

\section{Background}

Mucormycosis (Zygomycosis, phycomy- cosis) is an acute opportunistic infection caused by a saprophytic fungus that belongs to the class of phycomycetes. It is an aggressive, frequently fatal invasive fungal infection that can develop in immunocompromised patients and it is usually documented in patients with uncontrolled diabetes. Early diagnosis is of prime importance in treating patients with mucormycosis.

\section{Research frontiers}

Extraoral examination, radiographic findings, tissue biopsy with histopathology and pus culture were the diagnostic techniques for Mucormycosis of oral cavity. Early diagnosis and medical management included antifungal treatment were important in this case.

\section{Related reports}

Mucor is recognized as a triad of symptoms, such as uncontrolled diabetes mellitus, periorbital infection and meningoenchephalitis. Correcting or controlling predisposing Mucormycosis problem is essential for improving the treatment outcome. The other opportunistic fungal infection should be studied and reported in the other disease too.

\section{Innovations and breakthroughs}

Early diagnosis and medical management included antifungal treatment were important in this case. A proper understanding of such a fulminate entity is needed as the underlying systemic conditions at many times may be quiescent and dentists may be the first person to identify the debilitated status of the patient.

\section{Applications}

It may apply for the treatment in the other opportunistic fungal infection.

\section{Peer review}

This is a good case report about mucormycosis in diabetic ketoacidosis patient. It may apply for the treatment in the other opportunistic fungal infection.

\section{References}

[1] Fogarty C, Regennitter F, Viozzi CF. Invasive fungal infection of the maxilla following dental extractions in a patient with chronic obstructive pulmonary disease. J Can Dent Assoc 2006; 72(2): 149-152.

[2] Breiman A, Sadawsky D, Friedman J. Mucormycosis discussion and report of a case involving the maxillary sinus. Oral Surg Oral Med Oral Pathol 1981; 52: 375-378.

[3] Auluck A. Maxillary necrosis by mucormycosis. A case report and literature review. Med Oral Patol Oral Cir Bucal 2007; 12: 360-364.

[4] Moro JGM, Calleja JMLA, Garcia MB, Carretero JLC, Rodriguez JG. Rhinoorbitocerebral mucormycosis: A case report and literature review. Med Oral Patol Oral Cir Bucal 2008; 13(12): 792-795.

[5] Denning DW, Wilson GE. Fungal infections. In: The granulomatous disorders. James DG, Zumla A, editors. Cambridge, UK: Cambridge University Press; 1999, p. 235-256.

[6] Tugsel Z, Sezer B, Akalin T. Facial swelling and palatal ulceration in a diabetic patient. Oral Surg Oral Med Oral Pathol Oral Radiol Endod 2004; 98: 630-636.

[7] Petrikkos G, Skiada A, Lortholary O, Roilides E, Walsh T, Kontoyiannis DP. Oral epidemiology and clinical manifestations of mucormycosis. Clin Infect Dis 2012; 54: S23-34.

[8] Sun HY, Singh N. Mucormycosis: its contemporary face and management strategies. Lancet Infect Dis 2011; 11: 301-311.

[9] Lador N, Polacheck I, Gural A, Sanatski E, Garfunkel A. A trifungal infection of the mandible: Case report and literature review. Oral Surg Oral Med Oral Pathol 2006; 101: 451-456.

[10] Papadogeorgakis N, Eleni Parara E, Petsinis V, Vourlakou C. A case of successfully treated rhinocerebral mucormycosis: Dental implications. Int J Dent 2010. doi:10.1155/2010/273127.

[11] Ribeiro NF, Cousin GC, Wilson GE, Butterworth DM, Woodwards RT. Lethal invasive mucormycosis: Case report and recommendations for treatment. Int J Oral Maxillofac Surg 2001; 30: $156-159$

[12] Mathebula SD. Mucormycosis. S Afr Optom 2008; 67(1): 36-41.

[13] Jayachandran S, Krithika C. Mucormycosis presenting as palatal perforation. Indian J Dent Res 2006; 17: 139. 